\title{
POLYNOMIAL EXPANSION DETECTOR FOR UNIFORM LINEAR ARRAYS
}

\author{
Antonia Maria Masucci ${ }^{\star} \quad \varnothing y v i n d$ Ryan $^{\dagger} \quad$ Mérouane Debbah ${ }^{\star}$ \\ ${ }^{\star}$ Supélec, 3, rue Joliot-Curie, 91192 Gif-sur-Yvette, France \\ Email: \{antonia.masucci,merouane.debbah\}@supelec.fr \\ ${ }^{\dagger}$ Center of Mathematics for Applications, University of Oslo, \\ P.O. Box 1053 Blindern, NO-0316 Oslo, NORWAY \\ Email: oyvindry@ifi.uio.no
}

\begin{abstract}
In this paper, we design a low complexity linear MMSE decoder to recover the signal transmitted by $M$ mobile users to a base station equipped with $N$ receiving antennas, arranged as a uniform linear array (ULA). The angles of arrival are supposed to be uniformly distributed. As the dimension increases, it is hard to invert the system and therefore recent results on random matrix theory and polynomial expansion detectors are helpful to approximate the optimum linear MMSE receiver. Simulation results confirm the validity of this approximation.
\end{abstract}

Index Terms - Uniform Linear Array, Polynomial Receiver, Random Vandermonde Matrix

\section{INTRODUCTION}

In the last two decades, multiuser detection has shown to be a useful design technology for detecting desired signals from interference and noise. The first optimum multiuser detector was investigated by Verdú in [?] for asynchronous Gaussian multiple access channels based on maximum-likelihood detection. The receiver is considered optimal in the sense that the performance in the absence of Gaussian noise approaches that of a single-user system. Since the computation of the inverse matrix is complex, the authors of [2] introduced a multistage linear receiver to design different linear detectors, such as decorrelating detectors and minimum mean square error detector, approximating the inverse matrix by a polynomial expansion of the correlation matrix. This expansion is formed by a linear combination of the outputs of the individual receiver stages. The polynomial approximations are helpful in practice only if the weights can be calculated more easily than performing matrix inversion. As the optimum weights depend on the eigenvalues of the correlation matrix, they are not easy to calculate either. The combination of polynomial expansion detectors and convergence results from random matrix theory gives in [3] low-complexity detectors to mitigate interference in multiple-input multiple-output (MIMO) systems.

In this paper, we design a multiuser receiver using the linear minimum mean square error (MMSE) as a signal detector in a system model where $M$ mobile users are communicating in the uplink with a base station represented by a uniform linear array. The angles of arrival are supposed to be uniformly distributed and the channel matrix can be modeled by a random Vandermonde matrix. This scenario can be met in large surveillance system using radar. Using known

This work was supported by Alcatel-Lucent within the Alcatel-Lucent Chair on flexible radio at Supélec. The work of Antonia Maria Masucci was supported by Microsoft Research through its PhD Scholarship Programme. results on random matrix theory, in particular on the moments of random Vandermonde matrices with entries on the unit circle [4], we are able to find the optimum polynomial receiver, optimum with respect to the mean square error (MSE). Note that similar approaches could be used to find optimum receiver for compact rotating MIMO antenna [5]. In the next section, we present the model under observation. In section 3, we describe a low complexity linear MMSE receiver and in section 4 we use recent asynptotic results on Vandermonde matrices to estimate weights. In section 5, simulations show the validaty of the MMSE reciver design and in the last section we give the conclusions.

Notation. In the following, upper (lower) boldface symbols will be used for matrices (column vectors), whereas lower symbols will represent scalar values. $(.)^{H}$ will represent the hermitian transpose operator, $(.)^{T}$ denotes the transpose operator and $\|\cdot\|_{F}$ denotes the Frobenius norm operator. We denote by $\mathbf{I}_{M}$ the $M \times M$ identity matrix. We let $\operatorname{Tr}$ be the (non-normalized) trace for square matrices, defined by $\operatorname{Tr}(\mathbf{A})=\sum_{i=1}^{n} a_{i i}$, where $a_{i i}$ are the diagonal elements of the $M \times M$ matrix $\mathbf{A}$. We also let $\operatorname{tr}_{M}$ be the normalized trace, defined by $\operatorname{tr}_{M}(\mathbf{A})=\frac{1}{M} \operatorname{Tr}(\mathbf{A})$.

\section{SYSTEM MODEL}

We consider $M$ mobile users, each with a single antenna, communicating with a base station equipped with $N$ receiving antennas, arranged as a uniform linear array (ULA), as in Figure 1 . The $N \times 1$ output signal at the base station is given by

$$
\begin{aligned}
\mathbf{y}(t) & =\sum_{i=1}^{M} \mathbf{v}\left(\theta_{i}\right) p_{i}^{1 / 2} x_{i}(t)+\mathbf{n}(t) \\
& =\mathbf{V}(\theta) \mathbf{P}^{1 / 2} \mathbf{x}(t)+\mathbf{n}(t)
\end{aligned}
$$

where $\mathbf{x}$ is the $M \times 1$ input signal transmitted by the $M$ users

$$
\mathbf{x}(t)=\left[x_{1}(t), \ldots, x_{M}(t)\right]^{T},
$$

satisfying $\mathbb{E}\left[\mathbf{x}(t) \mathbf{x}(t)^{H}\right]=\mathbf{I}_{M}$, and $\mathbf{n}(t)$ is the additive Gaussian noise such that $\mathbb{E}\left[\mathbf{n}(t) \mathbf{n}(t)^{H}\right]=\sigma^{2} \mathbf{I}_{M}$. We suppose that the components in $\mathbf{x}(t)$ and $\mathbf{n}(t)$ are independent. The elements of the $M \times M$ matrix

$$
\mathbf{P}=\left[\begin{array}{cccc}
p_{1} & 0 & \cdots & 0 \\
0 & p_{2} & \cdots & 0 \\
\vdots & \ddots & \ddots & \vdots \\
0 & \cdots & 0 & p_{M}
\end{array}\right]
$$




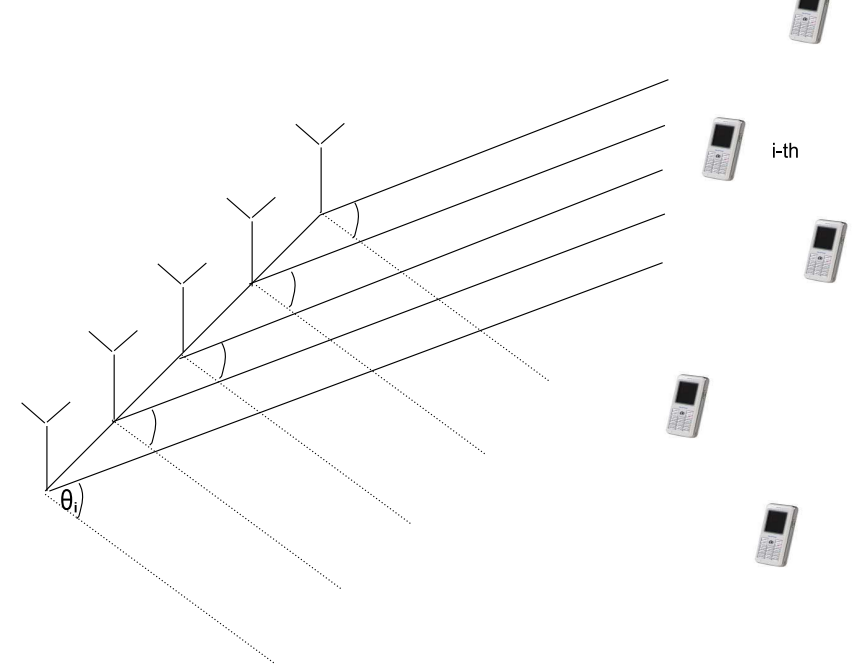

Fig. 1. Graphical representation of the system model.

represent the power with which the users send information. In the case of a line of sight between the mobile users and the base station, the $N \times M$ matrix $\mathbf{V}$ has the following form

$$
\mathbf{V}(\theta)=\frac{1}{\sqrt{N}}\left[\mathbf{v}\left(\theta_{1}\right), \ldots, \mathbf{v}\left(\theta_{M}\right)\right]
$$

where

$$
\mathbf{v}(\theta)=\left[1, e^{-j 2 \pi \frac{d}{\lambda} \sin (\theta)}, \ldots, e^{-j 2 \pi \frac{d}{\lambda}(N-1) \sin (\theta)}\right]^{T},
$$

and $\theta_{1}, \ldots, \theta_{M}$ are the angles of the users (see Figure 1) and are supposed to be i.i.d. and uniform on $]-\frac{\pi}{4}, \frac{\pi}{4}[, d$ is the interspacing distance between the antennas of the ULA, and $\lambda$ is the wavelenght of the signal. Assuming a memoryless channel, we can write

$$
\mathbf{y}=\mathbf{V} \mathbf{P}^{1 / 2} \mathbf{x}+\mathbf{n}
$$

\section{RECEIVER DESIGN}

The linear minimum mean square error (MMSE) detector [?] for the channel in (1) is given by

$$
\hat{\mathbf{x}}=\left(\mathbf{P}^{1 / 2} \mathbf{V}^{H} \mathbf{V} \mathbf{P}^{1 / 2}+\sigma^{2} \mathbf{I}\right)^{-1} \mathbf{P}^{1 / 2} \mathbf{V}^{H} \mathbf{y} .
$$

Recovering the data in (2), hence, requires the inversion of the matrix between brackets which is a difficult task when the dimensions of this matrix are large. In order to compute this, we remember the Caley-Hamilton theorem, which states that any square matrix satisfies its own characteristic equation

$$
\operatorname{det}\left(\mathbf{P}^{1 / 2} \mathbf{V}^{H} \mathbf{V} \mathbf{P}^{1 / 2}+\sigma^{2} \mathbf{I}_{M}-\left(\mu_{i}\left(p_{i}, \lambda_{i}\right)+\sigma^{2}\right) \mathbf{I}_{M}\right)=\mathbf{0},
$$

where $\lambda_{i}$ are the eigenvalues of $\mathbf{V}^{H} \mathbf{V}, p_{i}$ the eigenvalues of $\mathbf{P}$, and $\mu_{i}\left(p_{i}, \lambda_{i}\right)$ the eigenvalues of $\mathbf{P}^{1 / 2} \mathbf{V}^{H} \mathbf{V} \mathbf{P}^{1 / 2}$ (for large matrices, $\mu_{i}$ can be viewed as functions of $p_{i}, \lambda_{i}$, due to the almost sure convergence of products of Vandermonde matrices and deterministic matrices [7], which is why $\mu_{i}\left(p_{i}, \lambda_{i}\right)$ has been subsituted for the eigenvalues of $\mathbf{P}^{1 / 2} \mathbf{V}^{H} \mathbf{V} \mathbf{P}^{1 / 2}$ ). The characteristic polynomial can be written as

$$
\sum_{i=0}^{M} \alpha_{i}\left(\mathbf{P}^{1 / 2} \mathbf{V}^{H} \mathbf{V} \mathbf{P}^{1 / 2}+\sigma^{2} \mathbf{I}\right)^{i}=\mathbf{0}
$$

where $\alpha_{i}=\alpha_{i}\left(\lambda_{1}, \ldots, \lambda_{M} ; \sigma^{2} ; p_{1}, \ldots, p_{M}\right)$. We obtain

$$
\begin{aligned}
& \left(\mathbf{P}^{1 / 2} \mathbf{V}^{H} \mathbf{V} \mathbf{P}^{1 / 2}+\sigma^{2} \mathbf{I}\right)^{-1}= \\
& =-\sum_{i=1}^{M} \frac{\alpha_{i}}{\alpha_{0}}\left(\mathbf{P}^{1 / 2} \mathbf{V}^{H} \mathbf{V} \mathbf{P}^{1 / 2}+\sigma^{2} \mathbf{I}\right)^{i-1} \\
& =-\sum_{i=1}^{M} \frac{\alpha_{i}}{\alpha_{0}} \sum_{j=1}^{i-1}\left(\begin{array}{c}
i-1 \\
j
\end{array}\right) \sigma^{2(i-j-1)}\left(\mathbf{P}^{1 / 2} \mathbf{V}^{H} \mathbf{V} \mathbf{P}^{1 / 2}\right)^{i-1},
\end{aligned}
$$

which means that we can write the inverse as a matrix polynomial of degree $M-1$ in $\mathbf{P}^{1 / 2} \mathbf{V}^{H} \mathbf{V} \mathbf{P}^{1 / 2}$ such that

$$
\left(\mathbf{P}^{1 / 2} \mathbf{V}^{H} \mathbf{V} \mathbf{P}^{1 / 2}+\sigma^{2} \mathbf{I}\right)^{-1}=\sum_{i=0}^{M-1} \beta_{i}\left(\mathbf{P}^{1 / 2} \mathbf{V}^{H} \mathbf{V} \mathbf{P}^{1 / 2}\right)^{i},
$$

where the coefficients $\beta_{i}=\frac{\alpha_{i}}{\alpha_{0}} \sum_{j=1}^{i-1}\left(\begin{array}{c}i-1 \\ j\end{array}\right) \sigma^{2(i-j-1)}$ and they only depend on the eigenvalues of $\mathbf{V}^{H} \mathbf{V}$, the power $\mathbf{P}$ and the noise variance $\sigma^{2}$. In order to reduce the complexity, this suggests the introduction of multistage receivers that approximate the inverse matrix of the LMMSE receiver by a polynomial expansion of degree $K<M$ in $\mathbf{P}^{1 / 2} \mathbf{V}^{H} \mathbf{V} \mathbf{P}^{1 / 2}$ :

$$
\hat{\mathbf{x}}_{M M S E}=\sum_{i=1}^{K} \hat{\beta}_{i}\left(\mathbf{P}^{1 / 2} \mathbf{V}^{H} \mathbf{V} \mathbf{P}^{1 / 2}\right)^{i-1} \mathbf{P}^{1 / 2} \mathbf{V}^{H} \mathbf{y}
$$

The weight vector $\hat{\boldsymbol{\beta}}=\left[\hat{\beta}_{1}, \ldots, \hat{\beta}_{K}\right]^{T}$ can be choosen to optimize some performance measure. We derive the vector of weights such that minimizes the mean square error (MSE) of the estimated vector $\hat{\mathbf{x}}_{M M S E}$

$$
\hat{\boldsymbol{\beta}}=\arg \min _{\hat{\boldsymbol{\beta}}} \mathbb{E}\left[\left|\mathbf{x}-\sum_{i=1}^{K} \hat{\beta}_{i}\left(\mathbf{P}^{1 / 2} \mathbf{V}^{H} \mathbf{V} \mathbf{P}^{1 / 2}\right)^{i-1} \mathbf{P}^{1 / 2} \mathbf{V}^{H} \mathbf{y}\right|^{2}\right]
$$

Expressing equation (3) as a linear combination of the vectors $\mathbf{z}_{i}=$ $\left(\mathbf{P}^{1 / 2} \mathbf{V}^{H} \mathbf{V} \mathbf{P}^{1 / 2}\right)^{i-1} \mathbf{P}^{1 / 2} \mathbf{V}^{H} \mathbf{y}$, we can write the expectation in (4) as

$$
\mathbb{E}\left[|\mathbf{x}-\mathbf{Z} \hat{\boldsymbol{\beta}}|^{2}\right]
$$

where the matrix $\mathbf{Z}=\left[\mathbf{z}_{1}, \ldots, \mathbf{z}_{K}\right]$. We minimize respect to $\hat{\boldsymbol{\beta}}$ and we obtain that the optimum weight vector satisfies

$$
\hat{\boldsymbol{\beta}}=\left(\mathbb{E}\left[\mathbf{Z}^{H} \mathbf{Z}\right]\right)^{-1} \mathbb{E}\left[\mathbf{Z}^{H} \mathbf{x}\right]=\mathbf{\Phi}^{-1} \boldsymbol{\phi}
$$


The elements of the $K \times K$ matrix $\mathbf{\Phi}$ are given by

$$
\begin{aligned}
& \mathbf{\Phi}(i, j)=\mathbb{E}\left[\mathbf{z}_{i}^{H} \mathbf{z}_{j}\right] \\
& =\mathbb{E}\left[\left(\mathbf{x}^{H} \mathbf{P}^{1 / 2} \mathbf{V}^{H}+\mathbf{n}^{H}\right) \mathbf{V} \mathbf{P}^{1 / 2}\left(\mathbf{P}^{1 / 2} \mathbf{V}^{H} \mathbf{V} \mathbf{P}^{1 / 2}\right)^{i-1} \times\right. \\
& \left.\times\left(\mathbf{P}^{1 / 2} \mathbf{V}^{H} \mathbf{V} \mathbf{P}^{1 / 2}\right)^{j-1} \mathbf{P}^{1 / 2} \mathbf{V}^{H}\left(\mathbf{V} \mathbf{P}^{1 / 2} \mathbf{x}+\mathbf{n}\right)\right] \\
& =\frac{1}{M}\left[\operatorname{Tr}\left(\mathbf{P}^{1 / 2} \mathbf{V}^{H} \mathbf{V} \mathbf{P}^{1 / 2}\right)^{i+j}\right]+ \\
& +\frac{\sigma^{2}}{M}\left[\operatorname{Tr}\left(\mathbf{P}^{1 / 2} \mathbf{V}^{H} \mathbf{V} \mathbf{P}^{1 / 2}\right)^{i+j-1}\right] \\
& =\frac{1}{M}\left[\operatorname{Tr}\left(\mathbf{P} \mathbf{V}^{H} \mathbf{V}\right)^{i+j}\right]+\frac{\sigma^{2}}{M}\left[\operatorname{Tr}\left(\mathbf{P} \mathbf{V}^{H} \mathbf{V}\right)^{i+j-1}\right]
\end{aligned}
$$

and the elements of the $K \times 1$ vector $\phi$ are

$$
\begin{aligned}
\phi(i) & =\mathbb{E}\left[\mathbf{z}_{i}^{H} \mathbf{x}\right] \\
& =\mathbb{E}\left[\left(\mathbf{x}^{H} \mathbf{P}^{1 / 2} \mathbf{V}^{H}+\mathbf{n}^{H}\right) \mathbf{V} \mathbf{P}^{1 / 2}\left(\mathbf{P}^{1 / 2} \mathbf{V}^{H} \mathbf{V} \mathbf{P}^{1 / 2}\right)^{i-1} \mathbf{x}\right] \\
& =\frac{1}{M}\left[\operatorname{Tr}\left(\mathbf{P}^{1 / 2} \mathbf{V}^{H} \mathbf{V} \mathbf{P}^{1 / 2}\right)^{i}\right]=\frac{1}{M}\left[\operatorname{Tr}\left(\mathbf{P} \mathbf{V}^{H} \mathbf{V}\right)^{i}\right] .
\end{aligned}
$$

\section{ASYMPTOTIC ANALYSIS}

We need to recall some results from random matrix theory. We approximate the optimum weights by using results on the asymptotic moments of random Vandermonde matrices [4]. In particular, we assume that the size of $\mathbf{V}$ grows to infinity while the ratio of the number of columns to rows tends to a limit $r>0$.

Definition 1. An $N \times M$ random Vandermonde matrix with unit entries has the form

$$
\mathbf{V}=\frac{1}{\sqrt{N}}\left[\begin{array}{ccc}
1 & \cdots & 1 \\
e^{-j \omega_{1}} & \cdots & e^{-j \omega_{M}} \\
\vdots & \cdots & \vdots \\
e^{-j(N-1) \omega_{1}} & \cdots & e^{-j(N-1) \omega_{M}}
\end{array}\right]
$$

where $\omega_{1}, \ldots, \omega_{M}$ are i.i.d. random variables.

We will denote by $\mathcal{P}(n)$ the set of all partitions of $\{1, \ldots, n\}$, and will write $W_{1}, \ldots, W_{r}$ for the blocks of a given $\rho \in \mathcal{P}(n)$.

Definition 2. For $\rho \in \mathcal{P}(n)$, we define

$$
\begin{aligned}
& K_{\rho, \omega, N}= \\
& \frac{1}{N^{n+1-|\rho|}} \int_{(0,2 \pi)|\rho|} \prod_{k=1}^{n} \frac{1-e^{j N\left(\omega_{b(k-1)}-\omega_{b(k)}\right)}}{1-e^{j\left(\omega_{b(k-1)}-\omega_{b(k)}\right)}} d \omega_{1} \ldots d \omega_{|\rho|},
\end{aligned}
$$

where $\omega_{W_{1}}, \ldots, \omega_{W_{|\rho|}}$ are i.i.d. (indexed by the blocks of $\rho$ ) with the same distribution of $\omega$ and where $b(k)$ is the block of $\rho$ which contains $k$. If the limit

$$
K_{\rho, \omega}=\lim _{N \rightarrow \infty} K_{\rho, \omega, N}
$$

exists, it is called a Vandermonde mixed moment expansion coefficient.
In [4], it has been proved that the limit in (8) exists if the density of $\omega$ is continous. The calculation is based on combinatorial computation using crossing partitions since the matrices are not free. We will need the following result [4]:

Theorem 1. Let $\omega$ be as in Section 2, i.e. $\omega=2 \pi \frac{d}{\lambda} \sin (\theta)$ with $\theta$ uniform on $]-\frac{\pi}{4}, \frac{\pi}{4}\left[\right.$. We have that all limits $K_{\rho, \omega}$ exist. Also,

$$
m_{n}=\lim _{N \rightarrow \infty} \mathbb{E}\left[\operatorname{tr}_{M}\left(\mathbf{P} \mathbf{V}^{H} \mathbf{V}\right)^{n}\right]=\sum_{\rho \in \mathcal{P}(n)} K_{\rho, \omega} r^{|\rho|-1} P_{\rho}
$$

when $\frac{M}{N} \rightarrow r>0, P_{n}=\lim _{N \rightarrow \infty} \operatorname{tr}\left(\mathbf{P}^{n}\right), P_{\rho}=\prod_{i=1}^{k} P_{W_{i}}$.

The quantities (9) are also computable [8]. Using the previous result in Theorem 1, we obtain an estimation of the optimum weights for the considered model, when $N \rightarrow \infty, \frac{M}{N} \rightarrow r$, as follows

$$
\hat{\boldsymbol{\beta}}_{a s y}=\boldsymbol{\Phi}_{a s y}^{-1} \boldsymbol{\phi}_{a s y},
$$

where the generic element of $\boldsymbol{\Phi}_{a s y}$ is given by

$$
\begin{aligned}
\boldsymbol{\Phi}_{a s y}(i, j) & =\sum_{\rho \in \mathcal{P}(i+j)} K_{\rho, \omega} r^{|\rho|-1} P_{\rho}+\sigma^{2} \sum_{\rho \in \mathcal{P}(i+j-1)} K_{\rho, \omega} r^{|\rho|-1} P_{\rho} \\
& =m_{i+j}+\sigma^{2} m_{i+j-1}
\end{aligned}
$$

and

$$
\phi_{\text {asy }}(i)=\sum_{\rho \in \mathcal{P}(i)} K_{\rho, \omega} r^{|\rho|-1} P_{\rho}=m_{i} .
$$

This means that we can write the equation (10) in the following form

$$
\left[\begin{array}{c}
m_{1} \\
m_{2} \\
\vdots \\
m_{K}
\end{array}\right]=\left[\begin{array}{ccc}
m_{2}+\sigma^{2} m_{1} & \cdots & m_{K+1}+\sigma^{2} m_{K} \\
m_{3}+\sigma^{2} m_{2} & \cdots & m_{K+2}+\sigma^{2} m_{K+1} \\
\vdots & \vdots & \vdots \\
m_{K+1}+\sigma^{2} m_{K} & \cdots & m_{2 K}+\sigma^{2} m_{2 K-1}
\end{array}\right]\left[\begin{array}{c}
\hat{\beta}_{a s y}^{(1)} \\
\hat{\beta}_{a s y}^{(2)} \\
\vdots \\
\hat{\beta}_{a s y}^{(K)}
\end{array}\right]
$$

Note that we have substituted $\mathbb{E}\left[\operatorname{tr}\left(\mathbf{P} \mathbf{V}^{H} \mathbf{V}\right)^{n}\right]$ with $\left[\operatorname{tr}\left(\mathbf{P} \mathbf{V}^{H} \mathbf{V}\right)^{n}\right]$, which is valid due to almost sure convergence [7].

\section{NUMERICAL RESULTS}

In this section, we present simulations of the above result. Simulations show the validity of our approximation. Documentation of code for computing the moments of Vandermonde matrices can be found in [8].

In the Figure 2, we have plotted, increasing $M$ and $N$, the error $\left\|\mathbf{G}-\mathbf{G}_{a s y}\right\|_{F}^{2}$ where $\mathbf{G}=\left(\mathbf{P}^{1 / 2} \mathbf{V}^{H} \mathbf{V} \mathbf{P}^{1 / 2}+\sigma^{2} \mathbf{I}\right)^{-1} \mathbf{P}^{1 / 2} \mathbf{V}^{H}$ and $\mathbf{G}_{a s y}=\sum_{i=1}^{K} \hat{\beta}_{a s y}^{(i)}\left(\mathbf{P}^{1 / 2} \mathbf{V}^{H} \mathbf{V} \mathbf{P}^{1 / 2}\right)^{i-1} \mathbf{P}^{1 / 2} \mathbf{V}^{H}$. The ratio between the increasing $\mathrm{M}$ mobile users and $\mathrm{N}$ receiving antennas is assumes to be equal to $1 / 5,1,7 / 5$. In Figure $3,\|\mathbf{G}\|_{F}^{2}$ and $\left\|\mathbf{G}_{a s y}\right\|_{F}^{2}$ are plotted with increasing $M$ and $N$ with ratio $1 / 5$, and with approximation order $K=5$.

In Figure 5, we have plotted the SINR per user, increasing the number of receiving and mobile users with given ratio equal to $1 / 5,1,7 / 5$. In particular, the SINR of user $\ell(\ell=1, \ldots, M)$ reads $\operatorname{SINR}_{\ell}=\frac{\left|\mathbf{g}_{\ell} \mathbf{h}_{\ell}^{H}\right|^{2}}{\sum_{i=1, i \neq \ell}^{M}\left|\mathbf{g}_{\ell} \mathbf{h}_{i}^{H}\right|^{2}+\sigma^{2}}$ where $\mathbf{g}_{\ell}$ is the $\ell$-th row of the matrix $\mathbf{G}_{a s y}$ and $\mathbf{h}_{\ell}$ is the $\ell$-th column of the matrix product $\mathbf{P}^{1 / 2} \mathbf{V}$. Figure 4 shows good results for order of approximation $K=4$. In all simulations, we assume the power $\mathbf{P}=\mathbf{I}_{M}$, variance noise $\sigma=\sqrt{0.5}$, wavelenght $\lambda=2$, and distance between receiving antennas $d=1$. 


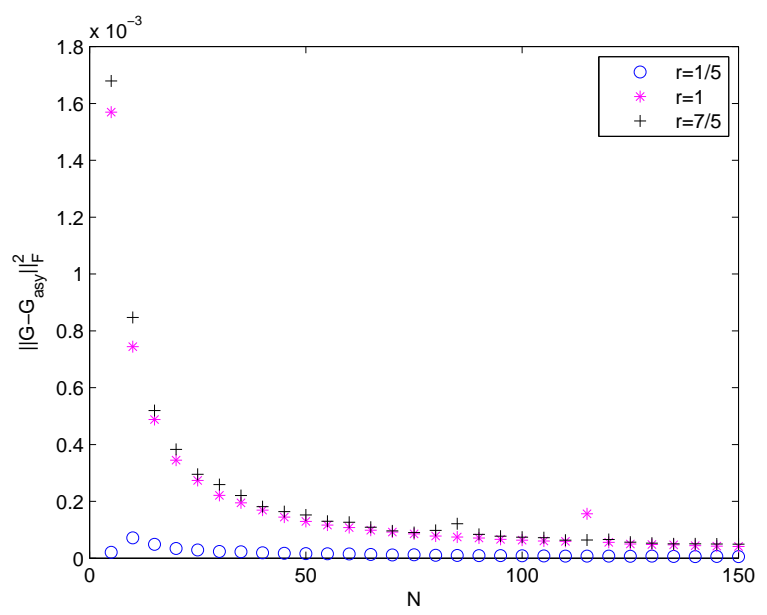

Fig. 2. $\left\|\mathbf{G}-\mathbf{G}_{a s y}\right\|_{F}^{2}$ is plotted increasing $M$ and $N$ with ratio $1 / 5,1,7 / 5$.

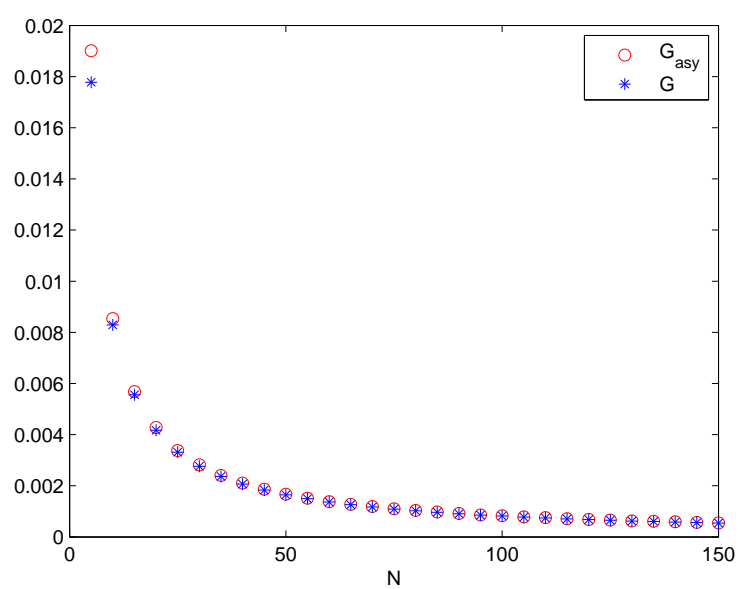

Fig. 3. $\|\mathbf{G}\|_{F}^{2}$ and $\left\|\mathbf{G}_{a s y}\right\|_{F}^{2}$ are plotted increasing $M$ and $N$ with ratio $1 / 5$.

\section{CONCLUSION}

In this paper, we have designed a low-complexity linear MMSE receiver through random matrix theory and polynomial expansion detectors. In particular, we have used recent asymptotic results on the moments of random Vandermonde matrices with entries on the unit circle to approximate the optimum weights of the polynomial receiver. Simulation results are presented in order to confirm the validity of our approximation.

\section{REFERENCES}

[1] S. Verdú, "Minimum probability of error for asynchronous gaussian multiple-access channels," IEEE Transactions on Information Theory, vol. 32, no. 1, pp. 85-96, 1986.

[2] S. Moshavi, E. G. Kanterakis, and D. L. Schilling, "Multistage

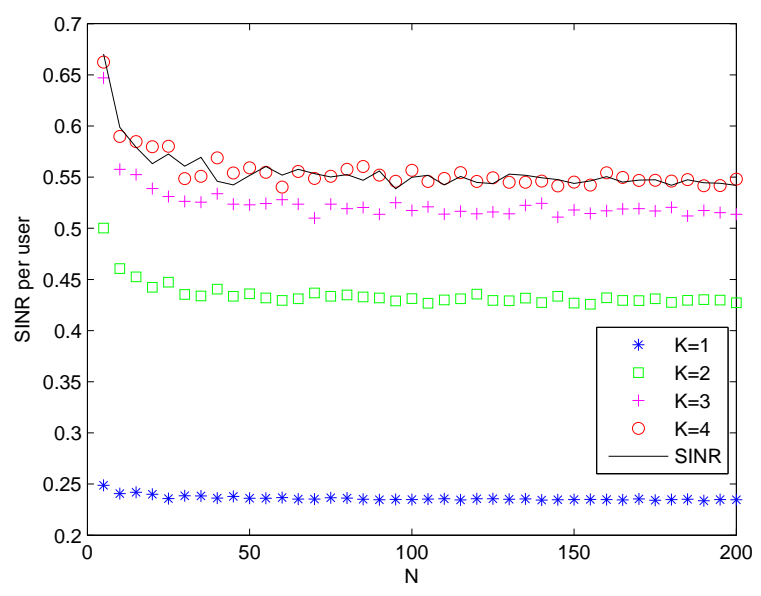

Fig. 4. SINR per user is plotted for $K=1,2,3,4$ increasing $M$ and $N$ with a ratio $3 / 5$.

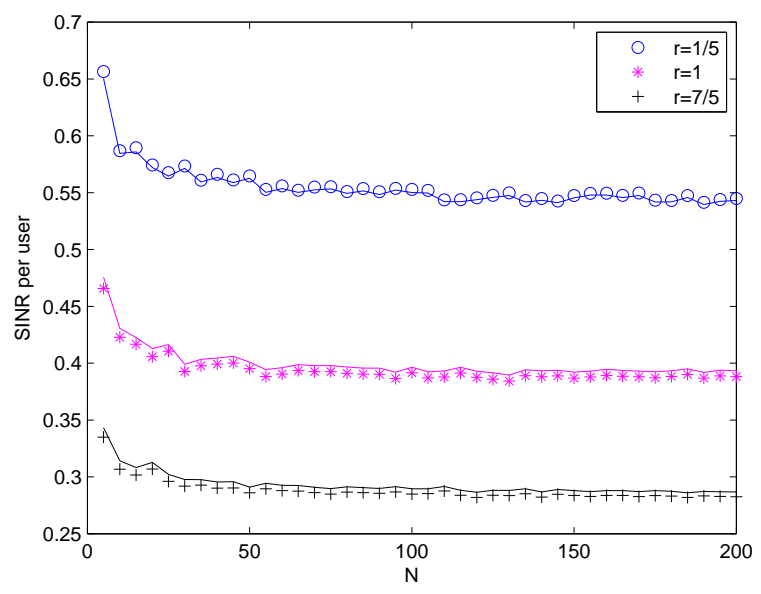

Fig. 5. SINR per user is plotted increasing $M$ and $N$ with a ratio $1 / 5,1,7 / 5$.

linear receivers for ds-cdma systems," Int. J. Wireless Inform. Networks, vol. 3, pp. 1-17, January 1996.

[3] R. Muller and S. Verdú, "Design and analysis of low-complexity interference mitigation on vector channels," Aug. 302001.

[4] Ø. Ryan and M. Debbah, "Asymptotic behaviour of random vandermonde matrices with entries on the unit circle," 2009.

[5] R. Bains and R. Muller, "On sampling issues of a virtually rotating mimo antenna," International ITG / IEEE Workshop on Smart Antennas (WSA), Reisensburg, Germany, 2006.

[6] S. Verdú, "Multiuser detection," Cambridge University Press, 1998.

[7] Ø. Ryan and M. Debbah, "Convolution operations arising from Vandermonde matrices," Submitted to IEEE Trans. on Information Theory, 2009.

[8] Ø. Ryan, "Documentation for the random matrix library," http://folk.uio.no/oyvindry/rmt/doc.pdf, 2009. 